\title{
3 Research Suare \\ The Effect of Cellulose Particles with Different Structure on the Stability of Thyme Oil Emulsion
}

Fuge Niu ( $\nabla$ niufg123@hotmail.com )

Zhejiang Gongshang University

Bin Zhang

Zhejiang Gongshang University

Yixuan Du

Zhejiang Gongshang University

Demei Hu

Zhejiang Gongshang University

Shuang Ma

Zhejiang Gongshang University

Weichun Pan

Zhejiang Gongshang University

\section{Research Article}

Keywords: Cellulose particle, Different structure, Thyme oil emulsion, Stability,

Posted Date: June 22nd, 2021

DOI: https://doi.org/10.21203/rs.3.rs-463464/v1

License: (c) (i) This work is licensed under a Creative Commons Attribution 4.0 International License.

Read Full License 


\section{Abstract}

The thyme oil emulsion was prepared using a novel type of spherical nanocellulose obtained under different hydrolysis time. The effect of different nanocellulose structures on the interfacial adsorption properties of emulsion and loading efficiency of thyme oil were analyzed. The results showed the particle size of emulsion decreased with the increasing of hydrolysis time. The loading efficiency of all emulsions for thyme oil reached about $80 \%$. The retention rate of thyme oil decreased during the storage period, and rising temperatures will exacerbate the loss of thyme oil. Compared to the $2 \mathrm{~h}$, emulsions stabilized by 10 $\mathrm{h}$ nanocellulose exhibited better stability and higher retention at all storage conditions. This was mainly due to the fact the nanocellulose $(10 \mathrm{~h})$ formed a dense structure that prevented the essential oils from volatilizing and migrating. The experimental results confirmed that cellulose can be used as stabilizer to encapsulate and transport hydrophobic active ingredient.

\section{Introduction}

In recent years, aromatic plants and their extracts have been examined for their effectiveness for food safety and preservation applications (Prakash, Kedia, Mishra, \& Dubey, 2015) and have received attention as growth and health promoters (Brenes \& Roura, 2010). Most of their properties are due to their essential oils and other secondary plant metabolite components. Phytochemicals, such as essential oils, contain a complex mixture of nonvolatile and volatile compounds produced by aromatic plants as secondary metabolites which have antioxidant, antiradical, and antimicrobial properties (Bakkali, Averbeck, Averbeck, \& Idaomar, 2008), and are classified by the United States Food and Drug Administration as generally recognized as safe (GRAS) (Weiss, Gaysinsky, Davidson, \& McClements, 2009). They have been widely used as functional ingredients in food, cosmetic, and pharmaceutical applications (Cimanga et al., 2002). For example, thyme oil has been shown to have inhibitory activities against various bacteria and yeasts (Gaysinsky, Davidson, McClements, \& Weiss, 2008). However, direct incorporation of essential oils in food systems encounters many challenges due to their low water solubility and interactive binding with food components such as protein and lipids, which limits their utilization in aqueous-based foods and beverages.

In order to expand the use of essential oils, a feasible way to improve essential oils dispersibility was to encapsulate essential oil within emulsion-based delivery systems. Emulsions are unstable system that consist of at least two incompatible liquids, whereby one of these liquids is dispersed in the other liquid in droplet-form. Emulsions tend to decompose over time through various occurrences, such as flocculation, coalescence, partial coalescence, or Ostwald ripening (Robins, 2000). Therefore, emulsifiers have been employed to promote the formation of dispersions by reducing the interfacial tension, and to prevent coalescence and flocculation of droplets by creating a net repulsion between the droplets (Dickinson). Generally, these emulsifiers can be broadly classified into three types: (i) Small molecular emulsifiers such as phospholipid (Pichot, Watson, \& Norton, 2013), and Saponins (Gutiérrez et al., 2008); (ii) biological macromolecular emulsifiers such as proteins (Joshi et al., 2012), polysaccharides (Xiangyang Li, Al-Assaf, Fang, \& Phillips, 2012), and starch (Charoen et al., 2011); (iii) colloidal particles, such as 
protein aggregates (Liu \& Tang, 2013), and protein/polysaccharide colloidal particles (Santos, Calero, Guerrero, \& Muñoz, 2015). In these emulsifiers, cellulose has had received considerable scholarly attention. Cellulose obtained via physical and chemical methods has attracted increasing interest in the fields of food, pharmaceutics, energy and chemicals research

most widespread and abundant natural biological resources, but also it has superior properties (including biocompatibility, biodegradable, nanoscale effect, high strength and strong surface activity) compared with inorganic nanoparticles. Cellulosic materials can also be hydrophobized to form food grade emulsions. Cellulose nanofibrils, cellulose nanocrystals, modified cellulose (TEMPO oxidized cellulose, OSA-modified cellulose) were used to reduce the lipid oxidation rate and improved

the stability of functional component (Chen et al., 2018; Kargar, Fayazmanesh, Alavi, Spyropoulos, \& Norton, 2012; Lu, Zhang, Li, \& Huang, 2018; Zhou et al., 2018). But the mean particle diameter of emulsions reached a few microns or even a dozen microns, and the emulsions showed varying degree of instability. Meanwhile, the essential oil had good solubility in the aqueous phase it often induced Ostwald ripening. Thus, how to use cellulose particles to prepare nanoscale essential oil emulsions, promoted their stability and improved their bioavailability, that was a new challenge.

In this study, the thyme oil emulsion was prepared using a novel type of spherical cellulose suspension. The effect of different cellulose structures on the interfacial adsorption properties of emulsion and loading efficiency and retention rate of thyme oil were analyzed. The objective was to reveal the relationship between cellulose structure and the adsorption properties at oil-in-water interface. These results could provide useful data for the stability and transportation of essential oils.

\section{Materials And Methods}

\subsection{Materials}

Microcrystalline cellulose was provided by Hangzhou Yuhan Technology Co., Ltd. (Hangzhou, China). Commercial sunflower oil was purchased from a local supermarket and contained approximately 13.0, 26.0 , and $61.0(\mathrm{w} / \mathrm{w})$ of saturated, monounsaturated, and polyunsaturated fats, respectively. Hydrochloric acid $(\mathrm{HCl}, 37 \% \mathrm{w} / \mathrm{w})$ and sulfuric acid $\left(\mathrm{H}_{2} \mathrm{SO}_{4}, 98 \% \mathrm{w} / \mathrm{w}\right)$ were purchased from Sinopharm Chemical Reagent Co., Ltd. (Shanghai, China). Thyme oil were purchased from Shanghai Lichen Biotechnology Co., Ltd (Shanghai, China).

\subsection{Preparation of cellulose}

Cellulose was prepared according to our previous reported method (Xin Li, Li, Shen, Niu, \& Fu, 2016). The mixed acid solution was prepared with sulfuric acid and hydrochloric acid ratio of 3:1 ( $\mathrm{v} / \mathrm{v})$, and then diluted with distilled water to obtain $55 \%$ mixed acid solution. Cellulose was prepared by the method ultrasonic-assisted acid hydrolysis and $8 \mathrm{~g}$ of microcrystalline cellulose was weighed. $100 \mathrm{~mL}$ of mixed acid solution was added and hydrolyzed under ultrasonic treatment at $100 \mathrm{~W}$ power (KQ-50DE, Lubo 
Instruments, China, Kunshan, China). The cellulose samples were hydrolyzed into 2, 6, 10 h. After hydrolysis, the hydrolysate was standing and cooled to room temperature. The sample was washed with distilled water and centrifuged at $8000 \mathrm{~g}$ for $10 \mathrm{~min}$, and repeated twice. The prepared cellulose nanoparticles with different morphology were stored at $4^{\circ} \mathrm{C}$.

\subsection{Morphology observation}

Based on the reported method (Wang, Ding, \& Cheng, 2007), the shape and size of both MCC and CNC samples were investigated by transmission electron microscopy (TEM, JEM-100CX II). The cellulose suspensions were diluted by distilled water to about $0.1 \%$ concentration and sonicated for 3 min. One drop of suspension was transferred on a copper grid, dried for $24 \mathrm{~h}$ at room temperature and then observed by TEM at $100 \mathrm{kV}$.

\subsection{Preparation of thyme oil emulsion}

According to our previous methods with minor modification (Niu et al., 2015). The emulsions were prepared by dispersing $1 \%(\mathrm{v} / \mathrm{v})$ thyme oil in cellulose solution at different hydrolysis duration. Primary emulsions were prepared using an Ultra-Turrax blender (IKA T25 Basic, Staufen, Germany) at 11,000 rpm for 2 min. Fine emulsions were prepared by twice homogenization with a high-pressure homogenizer at $40 \mathrm{MPa}$ using the homogenizer (APV1000, APV Co., Crawley, U.K.). Samples were sealed and stored at different temperature until further analysis.

\subsection{Particle size and ろ-potential analysis of thyme oil emulsion}

Based on the reported method (Niu et al., 2015), the emulsion was diluted 100 times with ultrapure water to avoid multiple light scattering. Then the emulsion was placed in the instrument before balancing the 60 s, and Mean particle diameter was recorded by dynamic light scattering (DLS) using a Nano-ZS90

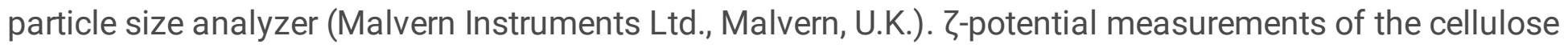
emulsion were performed by estimating the laser Doppler electrophoretic mobilities using a capillary electrophoresis cell (Zetasizer nano ZS, Malvern Instruments Ltd., Malvern, U.K.). The average value was taken for three times parallel measurement of each sample.

\subsection{In vitro digestion of thyme oil emulsion}

Simulated gastric fluid was prepared according to a previous study with minor modification (Yang \& McClements, 2013). Taken the emulsion sample $10 \mathrm{~mL}$ in the beaker and diluted 10 times using phosphoric acid buffer ( $5 \mathrm{mM}, \mathrm{pH} 7)$, the diluted emulsion was incubated in a water bath ( $\left.37^{\circ} \mathrm{C}\right)$ for 10 min, and then adjusted to $\mathrm{pH} 7$ using $\mathrm{NaOH}$ solution (range from 0.05 to $1 \mathrm{M}$ ).

The mixture was then incubated for $2 \mathrm{~h}$ at $37^{\circ} \mathrm{C}$ with simulated small intestinal fluid containing $5 \mathrm{~mL} 37.5$ $\mathrm{mg} / \mathrm{mL}$ bile salt, $1 \mathrm{~mL} 187.5 \mathrm{mM} \mathrm{CaCl}_{2}$ solution. Adjusting the mixed solution again to $\mathrm{pH} 7$ after fully mixed. Then $1.5 \mathrm{~mL}$ of $40 \mathrm{mg} / \mathrm{mL}$ pancreatic lipase was added into the mixed solution and. Since the amount of alkali solution that had to be added to the reaction chamber to maintain the $\mathrm{pH}$ at $7.0 \mathrm{was}$ 
recorded, and the release of free fatty acids was directly proportional to the amount of sodium hydroxide. So, the amount of alkali solution was used to determine the percentage of free fatty acids released from the system.

\subsection{Rheological properties of emulsion}

Rheological measurements of thyme oil emulsions were performed at $25^{\circ} \mathrm{C}$ using the rotary rheometer (MCR302, Antonopa, Austria) with a cone and plate geometry (cone diameter $=40 \mathrm{~mm}$, angle $=2^{\circ}, \mathrm{gap}=$ $0.05 \mathrm{~mm}$ ). For each measurement, $1.5 \mathrm{~mL}$ of the emulsion sample was loaded on the rheometer. The viscosity of emulsion was measured by a steady state flow program with the shear rate ranging from 0.1 to $1000 \mathrm{~s}^{-1}$ during $5 \mathrm{~min}$.

\subsection{Loading efficiency determination of thyme oil}

The loading efficiency of thyme oil was determined according to the method described by Zhong (Xue \& Zhong, 2014) with slightly modification: $50 \mu \mathrm{L}$ of emulsion were dissolved in $5 \mathrm{~mL}$ of $95 \mathrm{~mL} / 100 \mathrm{~mL}$ acetonitrile in water and mixed for $48 \mathrm{~h}$ in order to ensure that all the thyme oil active compound was released to solution. Before measurement, the solutions were centrifuged for $10 \mathrm{~min}$ at $6930 \mathrm{~g}$ and were filtered through $0.22 \mu \mathrm{m}$ Nylonfilters (VWR Intl., Radnor, PA., USA) to remove any insoluble substance from the solution. The optical density of solution was determined using an UV/vis spectrophotometer (WFJ 2000, UNICO, St. Louis, MO, USA) at $274 \mathrm{~nm}$. Each experiment was performed in duplicate. The average value of each sample was taken as the final result. The emulsification rate was calculated according to the formula:

Loading efficiency $(\%)=m_{1} / m_{2} \times 100 \%$

where, $\mathrm{m}_{1}$ is the amount of thyme oil trapped in the emulsion, and $\mathrm{m}_{2}$ is the total amount of thyme oil.

\subsection{Retention rate determination of thyme oil during storage}

The fresh emulsion was placed at room temperature for 7 days, and a certain amount of emulsion was regularly removed. According to the method of 2.7, the content of thyme oil was determined, and the retention rate of thyme oil was calculated according to the formula.

Retention rate $(\%)=\mathrm{m}_{3} / \mathrm{m}_{2} \times 100 \%$

where $m_{2}$ is the amount of thyme oil encapsulation, and $m_{3}$ is the residue amount of the thyme oil in the emulsion after storage.

\section{Results And Discussion}

\subsection{Particle size and morphology structure characterization}


In order to prepared stable emulsion, cellulose particles were produced using ultrasonic-assisted acid hydrolysis. Figure 2 shows the particle size of cellulose with different hydrolysis treatments as a function of aging time. Particle size of cellulose decreased as the hydrolysis time was increased. This was mainly because hydrogen ions produced by ionization of acid (hydrochloric and sulfuric acid) could destroy the cellulose glycosidic bonds of the molecular chain, resulting in a loss of non-crystalline region. Meanwhile, the energy, local high temperature, high pressure and cavitation effect produced by ultrasound can increase the surface area of cellulose, promote the diffusion of reagents, and result in the decrease of particle size. The TEM images of cellulose with different treatments are shown in Fig. 2. It was observed that the microcrystalline cellulose was damaged to sub-micrometer cellulose fragments with various shapes after being hydrolyzed for $2 \mathrm{~h}$. The average particle length of cellulose was about $500 \mathrm{~nm}$. Subsequently, it was clearly observed that the edges of the cellulose were broken, showing many irregular notches $(6 \mathrm{~h})$. As the hydrolysis time increased, the approximately axiolitic shape particles continuously formed, accompanied by a decrease in the micro-fragments. The particle size of cellulose was also reduced to less than $200 \mathrm{~nm}$.

\subsection{Stability analysis of thyme oil emulsion under different storage conditions}

In order to study the stability of thyme oil emulsion, the particle size change of thyme oil was analyzed during storage for 11 days at 4,25 and $37^{\circ} \mathrm{C}$, respectively. The mean particle size slightly increased during storage for 11 days at $4^{\circ} \mathrm{C}$ (Fig. 3a). For the cellulose at hydrolysis durations of $2 \mathrm{~h}$, the particle size of emulsion droplet was the largest and the particle increment rate was 7.89 compared with $6 \mathrm{~h}$

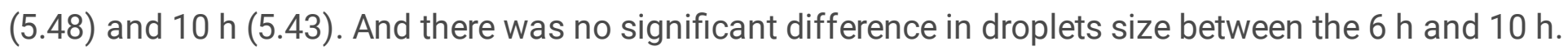
This was because the essential oils such as thyme oil have some degree of water solubility, promoting Ostwald ripening (Lim et al., 2011). The progressive decreased in emulsion size upon increasing hydrolysis durations have been widely observed for the emulsions stabilized by cellulose nanoparticles. This result suggested that granularity affects the relationship between the particle and particle-stabilized emulsions. In other words, the smaller particles cause the smaller emulsion droplet size (F. Niu, Pan, Su, \& Yang, 2016). The amorphous region of microcrystalline cellulose was destroyed after acid hydrolysis under ultrasonic condition (Guo, Guo, Wang, \& Yin, 2016). The cellulose particles formed under the ultrasonic crushing have higher specific surface area (Sovilj \& Petrović, 2006) and were more fully wrapped on the surface of thyme oil. Once they were attached to the oil water interface, they could produce irreversible adsorption, which could obstruct the droplet proximity in space and reduce the aggregation degree. On the other hand, it might be because cellulose particles formed a dense interface layer on the surface of oil droplets, which inhibited the violent aggregation between droplets.

At 25 and $37^{\circ} \mathrm{C}$, the increment rate of emulsion droplet size was significantly higher than that $4^{\circ} \mathrm{C}$. And the particle increment rate reached $22.27,20.19,17.81 \mathrm{for} 2,6,10 \mathrm{~h}$ at $37^{\circ} \mathrm{C}$, respectively. There results indicated that the storage temperature was the most important factor for the physical stability of emulsions. This may be because that the greater instability of emulsions with increased temperature was 
attributed to the loss of viscosity and the increased mobility of the system (Liang et al., 2012). However, in general, there was no obvious phase separation or emulsification stratification in the emulsion system at different 11 days storage time, indicating that the emulsion of thyme oil could maintain considerable stability in long time storage under certain conditions.

\subsection{Z-potential of thyme oil emulsion under different storage conditions}

The charge on the surface of emulsion droplet was also an important factor affecting the stability of emulsion. The $\zeta$-potential of thyme oil emulsion was investigated during storage for 11 days at different temperature (Fig. 4). The emulsion at $4^{\circ} \mathrm{C}$ carried more negative charge, and the negative charge on the surface of emulsion droplet becomes more and more with the decrease of particle size. This could be caused by two factors, 1) the negative charge of cellulose particles increased with the increasing of hydrolysis time, because cellulose particles dispersion had a large amount of negative charge due to more $0-\mathrm{SO}_{3}{ }^{-2}, \mathrm{C}_{-} \mathrm{SO}_{3}{ }^{-2}$, and $\mathrm{C}-\mathrm{OH}$ groups on cellulose particles surfaces and improved the stability of the cellulose emulsions (Xin Li et al., 2016). 2) more cellulose particles are attached to the surface of the oil droplets with increasing the hydrolysis durations, causing the accumulation of more negative charges. As the storage time increased, the $\zeta$-potential of emulsion decreased and there were significant changes in the early stages of storage. The possible hypothesis to explain the phenomenon was that the cellulose particles rearranged on the surface of the oil droplets and some particles desorbed from oil droplets. Because large oil droplets were dispersed through high energy in the initial homogenous process and then cellulose particles rapidly adsorbed to the surface of the oil droplets. However, the interface layer formed at the beginning was loose and irregular. Cellulose particles further combined with oil droplets and rearranged due to the hydrophobic and electrostatic interactions of cellulose particles. Finally, the cellulose particles formed a dense interface layer. Meanwhile, thyme oil contained relatively small nonpolar molecules with a relatively high water-solubility and its consists of large nonpolar triacylglycerol molecules with a very low water-solubility (F. Niu et al., 2016). Thus, the higher surface free energy leads to the formation of larger droplets to increase the stability of the system. In this process, part of a particle previously adsorbed may desorbed from the surface of the oil droplet, resulting in a decrease in total charge.

\subsection{Apparent viscosity change of thyme oil emulsion}

With the extension of hydrolysis time, we obtained different cellulose particles to prepare thyme oil emulsion. The results showed that the emulsion prepared by $2 \mathrm{~h}$ had higher viscosity (Fig. 5). The viscosity of emulsion decreased with increasing shear rate at low shear rates and showed Newtonian behavior. As the shear rate sufficiently increased to overcome the Brownian motion, the emulsion droplets became more ordered along the flow field and offered less resistance to flow and hence the lower viscosity (Sun, Gunasekaran, \& Richards, 2007). The viscosity at any test shear rate progressively decreased with increasing hydrolysis time from $2 \mathrm{~h}$ to $10 \mathrm{~h}$ and the emulsions stabilized by nanoparticles $(2 \mathrm{~h})$ had the highest viscosity, where it was attributed to the stable interfacial membrane and ordered spatial structure of emulsion. Nanoparticle produced a coated surfaces layer and such interfacial 
structure was sufficient to provide strong steric stabilization against flocculation/aggregation and coalescence. In the flow pattern, regular and dispersed emulsion droplets approaching another similar droplets altered its path to bypass the second droplets because of the steric hindrance and flocculation (ibanoğlu, 2002).

\subsection{Analysis of fat digestibility of thyme oil emulsion}

The influence of different nanoparticles on the free fatty acids release rate in the emulsions using the $\mathrm{pH}$ stat method was examined (Fig. 6). In general, there was a steep increase in the amount of free fatty acids released from the emulsions initially, followed by a more gradual increase at longer times. However, there were statistically significant differences between the rate and extent of digestion depending on the initial degree of dispersion of the oil droplets. In the case of the emulsions, the initial rate of digestion was much faster, and the final amount of free fatty acids released after $2 \mathrm{~h}$ of incubation was higher than for the pure sunflower oil. The main reason was that the oil droplet size in the emulsion system was small and uniform, and has a large specific surface area, which was conducive to the adsorption of lipase.

Other researchers have also found that the amount of free fatty acids produced per unit time increases as the oil droplet size decreases, which was attributed to changes in the surface area of oil exposed to the digestive enzymes (X.-M. Li et al., 2020). For the emulsion stabilized by cellulose particles, there were no significant difference in fatty acid fat release rate. This may be due to the fact that the emulsion droplets were very small and not very diverse, and were in full contact with the digestive enzymes, leading to similar rates of digestion. McClements et al. (2013) investigated influence of particle size on lipid digestion in emulsions and nanoemulsions, and found that small $\left(d_{32} 120 \mathrm{~nm}\right)$ and medium $\left(d_{32} 190\right.$ $\mathrm{nm}$ ) emulsions had the similar lipid digestion rate.

\subsection{Analysis of emulsification rate of thyme oil}

The application field was limited due to the volatility and instability of essential oil, consequently an appropriate emulsifier and formula are necessary to reduce the loss of functional material through embedding in the bilayer wall. Figure 7 shows the effect of cellulose particle with different hydrolysis time on loading efficiency of thyme oil. When the hydrolysis time was $2 \mathrm{~h}$, the loading efficiency of thyme oil reached $79 \%$. The loading efficiency of thyme oil slightly increased as the hydrolysis time increased. But, no significant change was observed in the loading efficiency of thyme oil under different hydrolysis time, indicating that the emulsifying effect of the cellulose obtained was the similar. Though the cellulose prepared under hydrolysis $10 \mathrm{~h}$ had lower wetting properties compared with $2 \mathrm{~h}$. It can be inferred that the cellulose particles prepared under hydrolysis durations $10 \mathrm{~h}$ could be easily adsorbed to the interface oil droplets. The most likely explanation for this result was that, at longer hydrolysis durations, the number of particles did not increase proportionately because the yield of cellulose decreased (Xin Li et al., 2016).

\subsection{Changes of retention rate of thyme oil during storage}


Thyme oil was a volatile essential oil substance, so the stability of thyme oil was an important parameter to measure the performance of emulsifier. 7 days storage was carried out to investigate the effect of temperature on the chemical stability of thyme oil emulsions (Fig. 8). The retention of thyme oil in samples at all the storage conditions decreased during the storage period. When the emulsion stored at $4^{\circ} \mathrm{C}$, the loss of thyme oil was less and the retention rate was still above $80 \%$ after 7 days. When the temperature rose to $25^{\circ} \mathrm{C}$ and $37^{\circ} \mathrm{C}$, the retention of thyme oil dropped to $70 \%$ and $55 \%$ for emulsion stabilized by cellulose $(2 \mathrm{~h})$, respectively. This was mainly because essential oils contained a complex mixture of nonvolatile and volatile compounds produced by aromatic plants as secondary metabolites. Increasing temperature will accelerate the evaporation of essential oils and the penetration of watersoluble components. The increase of temperature will accelerate the evaporation of essential oils and the penetration of water-soluble components, leading to the decrease of retention rate. Liang et al. (2013) found that $51.25 \%, 64.36 \%$ of $\beta$-carotene was retained in the starch emulsions at the end of 30 days when stored at $25^{\circ} \mathrm{C}$ and $4^{\circ} \mathrm{C}$, respectively. Compared to the $2 \mathrm{~h}$ cellulose particles, emulsions stabilized by $10 \mathrm{~h}$ cellulose particles exhibited improved stability and higher retentions at all storage conditions. This was mainly due to the fact that the cellulose $(10 \mathrm{~h})$ formed a dense viscoelastic structure that prevented the essential oils from volatilizing and migrating (Fig. 5). It was also further demonstrated that the emulsion prepared from cellulose particles formed by hydrolysis for $10 \mathrm{~h}$ had better stability.

\section{Conclusion}

The encapsulation of thyme oil with cellulose emulsion as carrier system was studied. The storage stability, loading efficiency and retention and of the emulsion were analyzed. The experimental results showed that when the cellulose concentration was $0.9 \%$, the thyme oil concentration was $1 \%$, the cellulose particles with different hydrolysis time can reach the loading efficiency of about $80 \%$. After the storage of 7 days, the droplet particle size increased from $400 \mathrm{~nm}$ to $600 \mathrm{~nm}$ at different temperatures.

The retention the retention rate was still above $80 \%$, and thyme oil emulsions stabilized by $10 \mathrm{~h}$ cellulose particles exhibited better stability and higher retentions at all storage conditions compared to the $2 \mathrm{~h}$ cellulose particles due to the dense interfacial structure. There was no significant phase separation and cream during the storage period, and showing good electrostatic stability. Compared to the pure sunflower oil, thyme oil emulsion had higher fat release rate. Cellulose emulsion can increase the dispersion and improve the stability of thyme oil. The experimental results can be used to embed and transport the active substances and provide data support for designing stable emulsion systems using cellulose particles with a different structure.

\section{Declarations}

\section{Ethical statements}

I certify that this manuscript is original and has not been published and will not be submitted elsewhere for publication while being considered by Cellulose. And the study is not split up into several parts to increase the quantity of submissions and submitted to various journals or to one journal over time. No 
data have been fabricated or manipulated (including images) to support your conclusions. No data, text, or theories by others are presented as if they were our own.

The submission has been received explicitly from all co-authors. And authors whose names appear on the submission have contributed sufficiently to the scientific work and therefore share collective responsibility and accountability for the results.

Conflict of Interest: The authors declare that they have no conflict of interest. This article does not contain any studies with human participants or animals performed by any of the authors. Informed consent was obtained from all individual participants included in the study."

\section{Acknowledgements}

The authors gratefully acknowledge financial supported by National Natural Science Foundation of China (31701650, 31171713), the Public welfare projects of Zhejiang province (2016C32011), the Natural Science Foundation of Zhejiang Province (LY17C200004), National Key Research and Development Program of China (2018YFD0400303).

\section{References}

1. Bakkali, F., Averbeck, S., Averbeck, D., \& Idaomar, M. (2008). Biological effects of essential oils-a review. Food and chemical toxicology, 46(2), 446-475.

2. Brenes, A., \& Roura, E. (2010). Essential oils in poultry nutrition: Main effects and modes of action. Animal feed science and technology, 158(1-2), 1-14.

3. Brinchi, L., Cotana, F., Fortunati, E., \& Kenny, J. M. (2013). Production of nanocrystalline cellulose from lignocellulosic biomass: Technology and applications. Carbohydrate Polymers, 94(1), 154-169.

4. Charoen, R., Jangchud, A., Jangchud, K., Harnsilawat, T., Naivikul, O., \& McClements, D. J. (2011). Influence of Biopolymer Emulsifier Type on Formation and Stability of Rice Bran Oil-in-Water Emulsions: Whey Protein, Gum Arabic, and Modified Starch. Journal of food science, 76(1), 165-172.

5. Chen, Q.-H., Zheng, J., Xu, Y.-T., Yin, S.-W., Liu, F., \& Tang, C.-H. (2018). Surface modification improves fabrication of pickering high internal phase emulsions stabilized by cellulose nanocrystals. Food Hydrocolloids, 75, 125-130.

6. Cimanga, K., Apers, S., de Bruyne, T., Van Miert, S., Hermans, N., Totté, J., Tona, L. (2002). Chemical composition and antifungal activity of essential oils of some aromatic medicinal plants growing in the Democratic Republic of Congo. Journal of Essential Oil Research, 14(5), 382-387.

7. Dickinson, E. Use of nanoparticles and microparticles in the formation and stabilization of food emulsions. Trends in Food Science \& Technology, 24(1), 0-12.

8. Gaysinsky, S., Davidson, P. M., McClements, D. J., \& Weiss, J. (2008). Formulation and characterization of phytophenol-carrying antimicrobial microemulsions. Food Biophysics, 3(1), 54- 
65.

9. Guo, J., Guo, X., Wang, S., \& Yin, Y. (2016). Effects of ultrasonic treatment during acid hydrolysis on the yield, particle size and structure of cellulose nanocrystals. Carbohydr Polym, 135, 248-255.

10. Gutiérrez, J. M., González, C., Maestro, A., Solè, I., Pey, C. M., \& Nolla, J. (2008). Nano-emulsions: New applications and optimization of their preparation. Current Opinion in Colloid \& Interface Science, 13(4), 245-251.

11. İbanoğlu, E. (2002). Rheological behaviour of whey protein stabilized emulsions in the presence of gum arabic. Journal of Food Engineering, 52(3), 273-277.

12. Joshi, M., Adhikari, B., Aldred, P., Panozzo, J., Kasapis, S., \& Barrow, C. (2012). Interfacial and emulsifying properties of lentil protein isolate. Food Chemistry, 134(3), 1343-1353.

13. Kargar, M., Fayazmanesh, K., Alavi, M., Spyropoulos, F., \& Norton, I. T. (2012). Investigation into the potential ability of Pickering emulsions (food-grade particles) to enhance the oxidative stability of oilin-water emulsions. J Colloid Interface Sci, 366(1), 209-215.

14. Li, X.-M., Li, X., Wu, Z., Wang, Y., Cheng, J.-S., Wang, T., \& Zhang, B. (2020). Chitosan hydrochloride/carboxymethyl starch complex nanogels stabilized Pickering emulsions for oral delivery of $\beta$-carotene: Protection effect and in vitro digestion study. Food Chemistry, 315, 126288.

15. Li, X., Al-Assaf, S., Fang, Y., \& Phillips, G. O. (2012). Competitive adsorption between sugar beet pectin (SBP) and hydroxypropyl methylcellulose (HPMC) at the oil/water interface. Carbohydrate Polymers, 91(2), 573-580.

16. Li, X., Li, K., Shen, Y., Niu, F., \& Fu, Y. (2016). Influence of pure gum on the physicochemical properties of whey protein isolate stabilized oil-in-water emulsions. Colloids and Surfaces A: Physicochemical and Engineering Aspects, 504, 442-448.

17. Liang, R., Shoemaker, C. F., Yang, X., Zhong, F., \& Huang, Q. (2013). Stability and bioaccessibility of $\beta$ carotene in nanoemulsions stabilized by modified starches. J Agric Food Chem, 61(6), 1249-1257.

18. Liang, R., Xu, S., Shoemaker, C. F., Li, Y., Zhong, F., \& Huang, Q. (2012). Physical and Antimicrobial Properties of Peppermint Oil Nanoemulsions. J Agric Food Chem, 60(30), 7548-7555.

19. Lim, S. S., Baik, M. Y., Decker, E. A., Henson, L., Popplewell, L. M., McClements, D. J., \& Choi, S. J. (2011). Stabilization of orange oil-in-water emulsions: a new role for ester gum as an Ostwald ripening inhibitor. Food Chemistry, 128(4), 1023-1028.

20. Liu, F., \& Tang, C.-H. (2013). Soy Protein Nanoparticle Aggregates as Pickering Stabilizers for Oil-inWater Emulsions. J Agric Food Chem, 61(37), 8888-8898.

21. Lu, X., Zhang, H., Li, Y., \& Huang, Q. (2018). Fabrication of milled cellulose particles-stabilized Pickering emulsions. Food Hydrocolloids, 77, 427-435.

22. Niu, Zhou, J., Niu, D., Wang, C., Liu, Y., Su, Y., \& Yang, Y. (2015). Synergistic effects of ovalbumin/gum arabic complexes on the stability of emulsions exposed to environmental stress. Food Hydrocolloids, 47, 14-20. 
23. Niu, F., Pan, W., Su, Y., \& Yang, Y. (2016). Physical and antimicrobial properties of thyme oil emulsions stabilized by ovalbumin and gum arabic. Food Chemistry, 212, 138-145.

24. Pichot, R., Watson, R. L., \& Norton, I. T. (2013). Phospholipids at the interface: current trends and challenges. International journal of molecular sciences, 14(6), 11767-11794.

25. Prakash, B., Kedia, A., Mishra, P. K., \& Dubey, N. (2015). Plant essential oils as food preservatives to control moulds, mycotoxin contamination and oxidative deterioration of agri-food commoditiesPotentials and challenges. Food Control, 47, 381-391.

26. Robins, M. M. (2000). Emulsions - creaming phenomena. Current Opinion in Colloid \& Interface Science, 5(5), 265-272.

27. Salvia-Trujillo, L., Qian, C., Martín-Belloso, O., \& McClements, D. J. (2013). Influence of particle size on lipid digestion and $\beta$-carotene bioaccessibility in emulsions and nanoemulsions. Food Chemistry, 141(2), 1472-1480.

28. Santos, J., Calero, N., Guerrero, A., \& Muñoz, J. (2015). Relationship of rheological and microstructural properties with physical stability of potato protein-based emulsions stabilized by guar gum. Food Hydrocolloids, 44(0), 109-114.

29. Sovilj, V. J., \& Petrović, L. B. (2006). Influence of hydroxypropylmethyl cellulose-sodium dodecylsulfate interaction on the solution conductivity and viscosity and emulsion stability. Carbohydrate Polymers, 64(1), 41-49.

30. Sun, C., Gunasekaran, S., \& Richards, M. P. (2007). Effect of xanthan gum on physicochemical properties of whey protein isolate stabilized oil-in-water emulsions. Food Hydrocolloids, 21(4), 555564.

31. Wang, N., Ding, E., \& Cheng, R. (2007). Thermal degradation behaviors of spherical cellulose nanocrystals with sulfate groups. Polymer, 48(12), 3486-3493.

32. Weiss, J., Gaysinsky, S., Davidson, M., \& McClements, J. (2009). Nanostructured encapsulation systems: food antimicrobials. Global issues in food science and technology, chapter 24, pp. 425-479.

33. Xue, J., \& Zhong, Q. (2014). Thyme Oil Nanoemulsions Coemulsified by Sodium Caseinate and Lecithin. J Agric Food Chem, 62(40), 9900-9907.

34. Yang, Y., \& McClements, D. J. (2013). Vitamin E bioaccessibility: Influence of carrier oil type on digestion and release of emulsified a-tocopherol acetate. Food Chemistry, 141(1), 473-481.

35. Zhou, Y., Sun, S., Bei, W., Zahi, M. R., Yuan, Q., \& Liang, H. (2018). Preparation and antimicrobial activity of oregano essential oil Pickering emulsion stabilized by cellulose nanocrystals. Int J Biol Macromol, 112, 7-13.

\section{Figures}




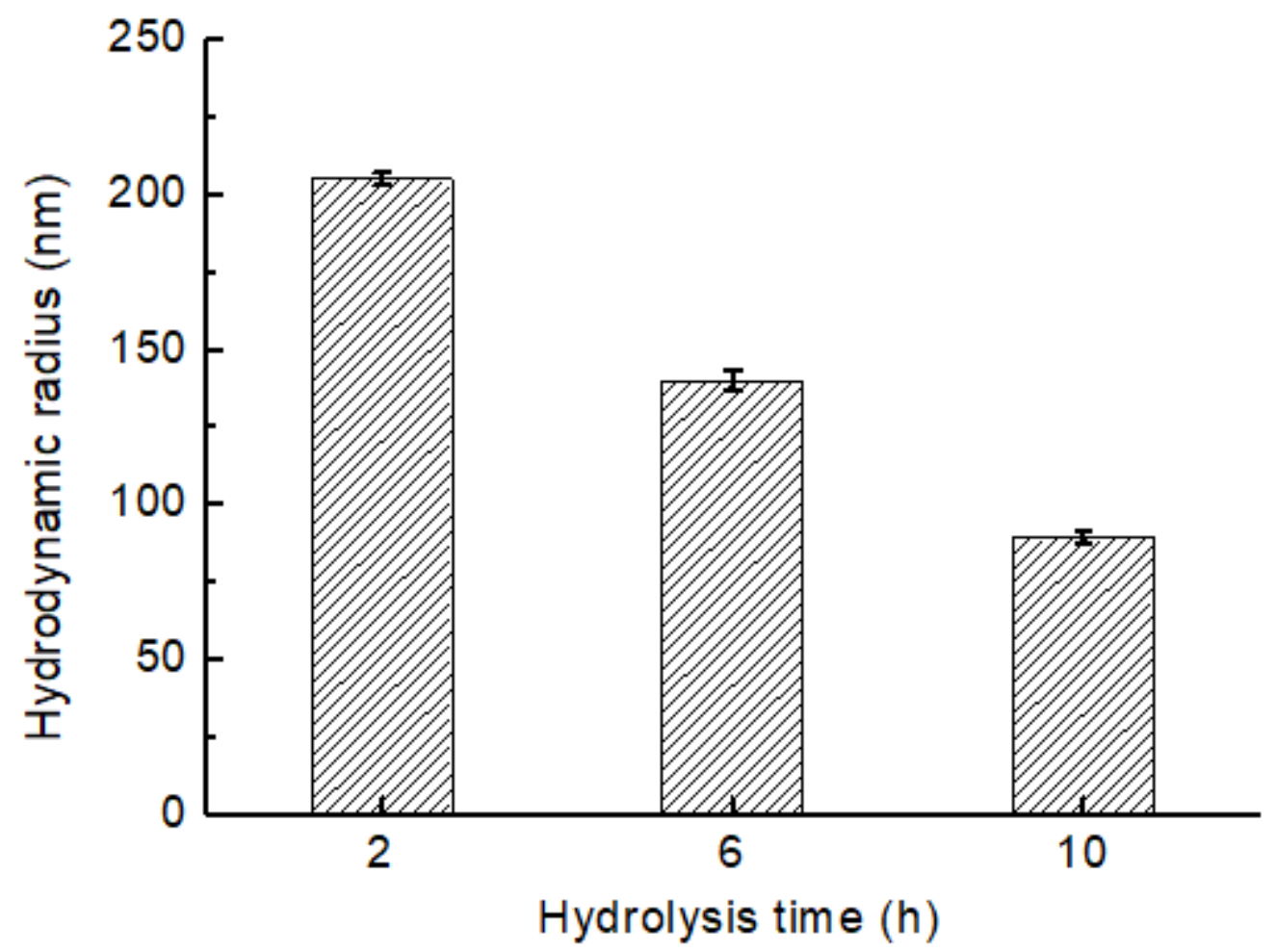

\section{Figure 1}

In order to prepared stable emulsion, cellulose particles were produced using ultrasonic-assisted acid hydrolysis. Fig. 1 shows the particle size of cellulose with different hydrolysis treatments as a function of aging time. Particle size of cellulose decreased as the hydrolysis time was increased. This was mainly because hydrogen ions produced by ionization of acid (hydrochloric and sulfuric acid) could destroy the cellulose glycosidic bonds of the molecular chain, resulting in a loss of non-crystalline region.
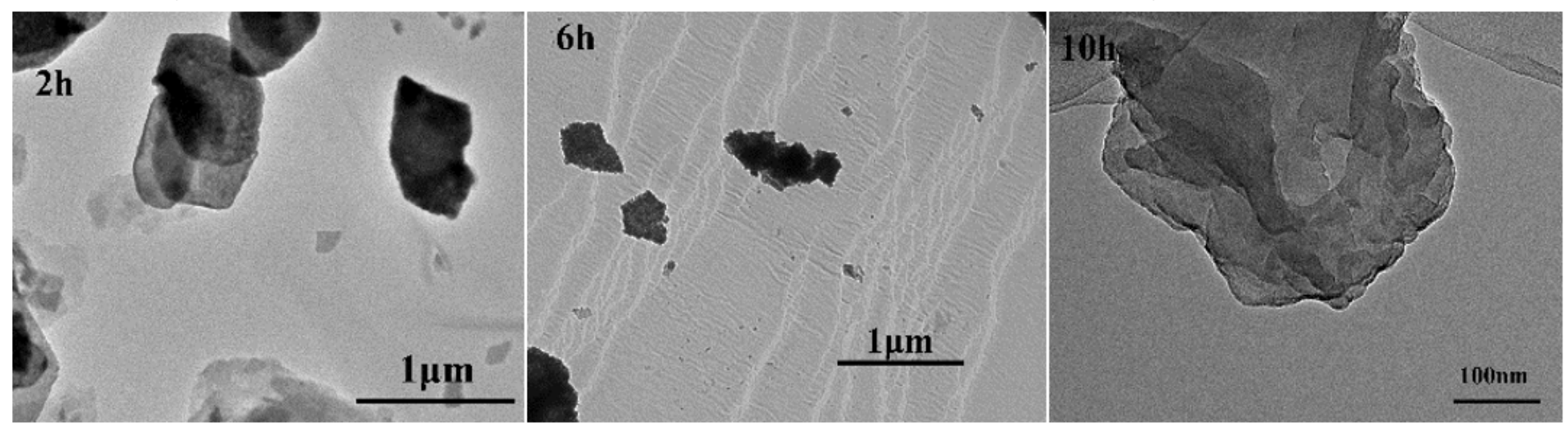

\section{Figure 2}

The TEM images of cellulose with different treatments are shown in Fig. 2. It was observed that the microcrystalline cellulose was damaged to sub-micrometer cellulose fragments with various shapes after being hydrolyzed for $2 \mathrm{~h}$. The average particle length of cellulose was about $500 \mathrm{~nm}$. Subsequently, it was clearly observed that the edges of the cellulose were broken, showing many irregular notches $(6 \mathrm{~h})$. As the 
hydrolysis time increased, the approximately axiolitic shape particles continuously formed, accompanied by a decrease in the micro-fragments. The particle size of cellulose was also reduced to less than 200 $\mathrm{nm}$.
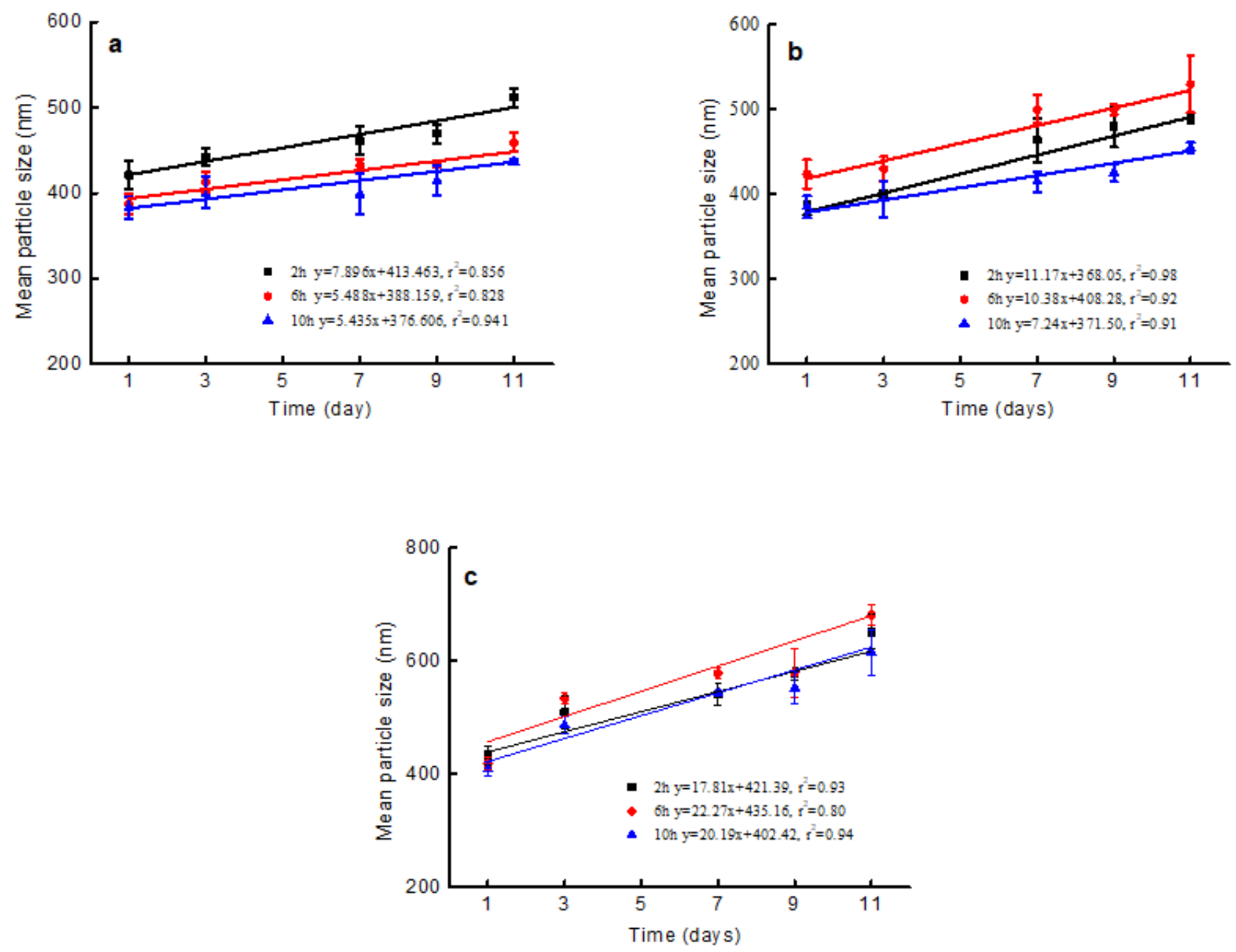

\section{Figure 3}

In order to study the stability of thyme oil emulsion, the particle size change of thyme oil was analyzed during storage for 11 days at 4, 25 and $370 \mathrm{C}$, respectively. The mean particle size slightly increased during storage for 11 days at $4 \mathrm{oC}$ (Fig. 3a). For the cellulose at hydrolysis durations of $2 \mathrm{~h}$, the particle size of emulsion droplet was the largest and the particle increment rate was 7.89 compared with $6 \mathrm{~h}$ (5.48) and $10 \mathrm{~h}$ (5.43). And there was no significant difference in droplets size between the $6 \mathrm{~h}$ and $10 \mathrm{~h}$. This was because the essential oils such as thyme oil have some degree of water solubility, promoting Ostwald ripening (Lim et al., 2011). The progressive decreased in emulsion size upon increasing hydrolysis durations have been widely observed for the emulsions stabilized by cellulose nanoparticles. 

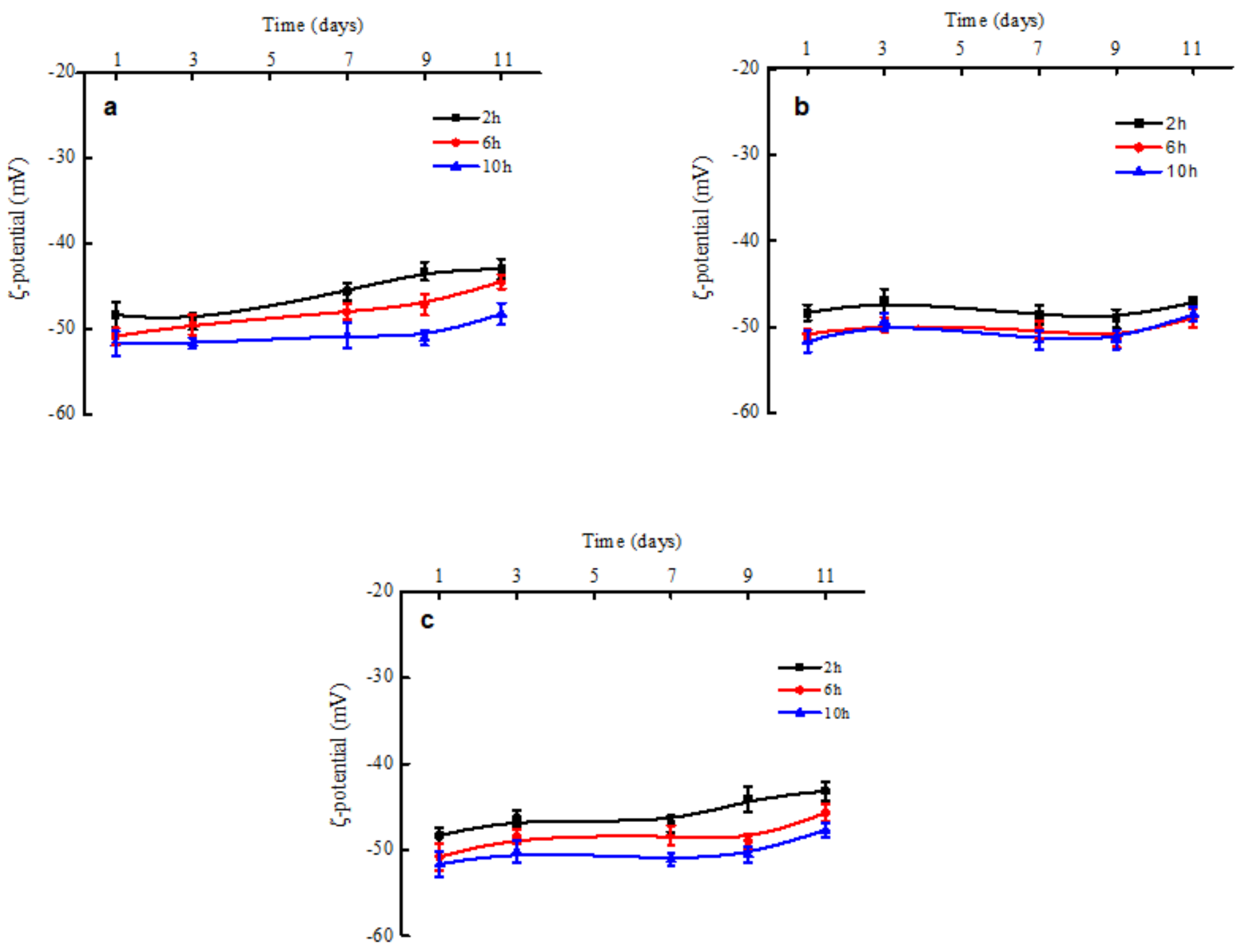

\section{Figure 4}

The charge on the surface of emulsion droplet was also an important factor affecting the stability of emulsion. The $\zeta$-potential of thyme oil emulsion was investigated during storage for 11 days at different temperature (Fig. 4). The emulsion at $4 \mathrm{oC}$ carried more negative charge, and the negative charge on the surface of emulsion droplet becomes more and more with the decrease of particle size. This could be caused by two factors, 1) the negative charge of cellulose particles increased with the increasing of hydrolysis time, because cellulose particles dispersion had a large amount of negative charge due to more $\mathrm{O}-\mathrm{SO} 3-2, \mathrm{C}-\mathrm{SO} 3-2$, and $\mathrm{C}-\mathrm{OH}$ groups on cellulose particles surfaces and improved the stability of the cellulose emulsions (Xin Li et al., 2016). 2) more cellulose particles are attached to the surface of the oil droplets with increasing the hydrolysis durations, causing the accumulation of more negative charges. As the storage time increased, the $\zeta$-potential of emulsion decreased and there were significant changes in the early stages of storage. 


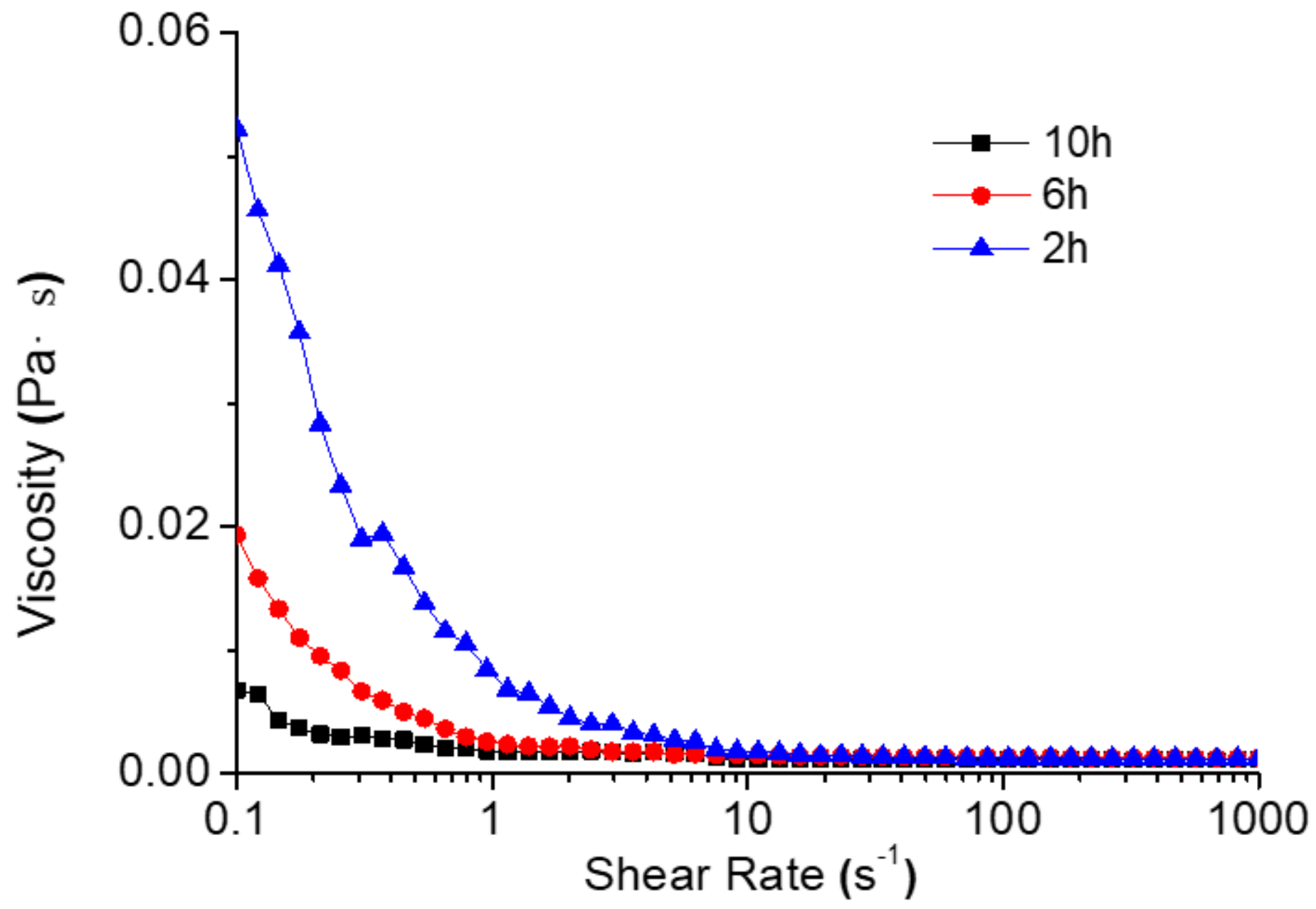

Figure 5

With the extension of hydrolysis time, we obtained different cellulose particles to prepare thyme oil emulsion. The results showed that the emulsion prepared by $2 \mathrm{~h}$ had higher viscosity (Fig. 5). The viscosity of emulsion decreased with increasing shear rate at low shear rates and showed Newtonian behavior. As the shear rate sufficiently increased to overcome the Brownian motion, the emulsion droplets became more ordered along the flow field and offered less resistance to flow and hence the lower viscosity (Sun, Gunasekaran, \& Richards, 2007). The viscosity at any test shear rate progressively decreased with increasing hydrolysis time from $2 \mathrm{~h}$ to $10 \mathrm{~h}$ and the emulsions stabilized by nanoparticles $(2 \mathrm{~h})$ had the highest viscosity, where it was attributed to the stable interfacial membrane and ordered spatial structure of emulsion. 


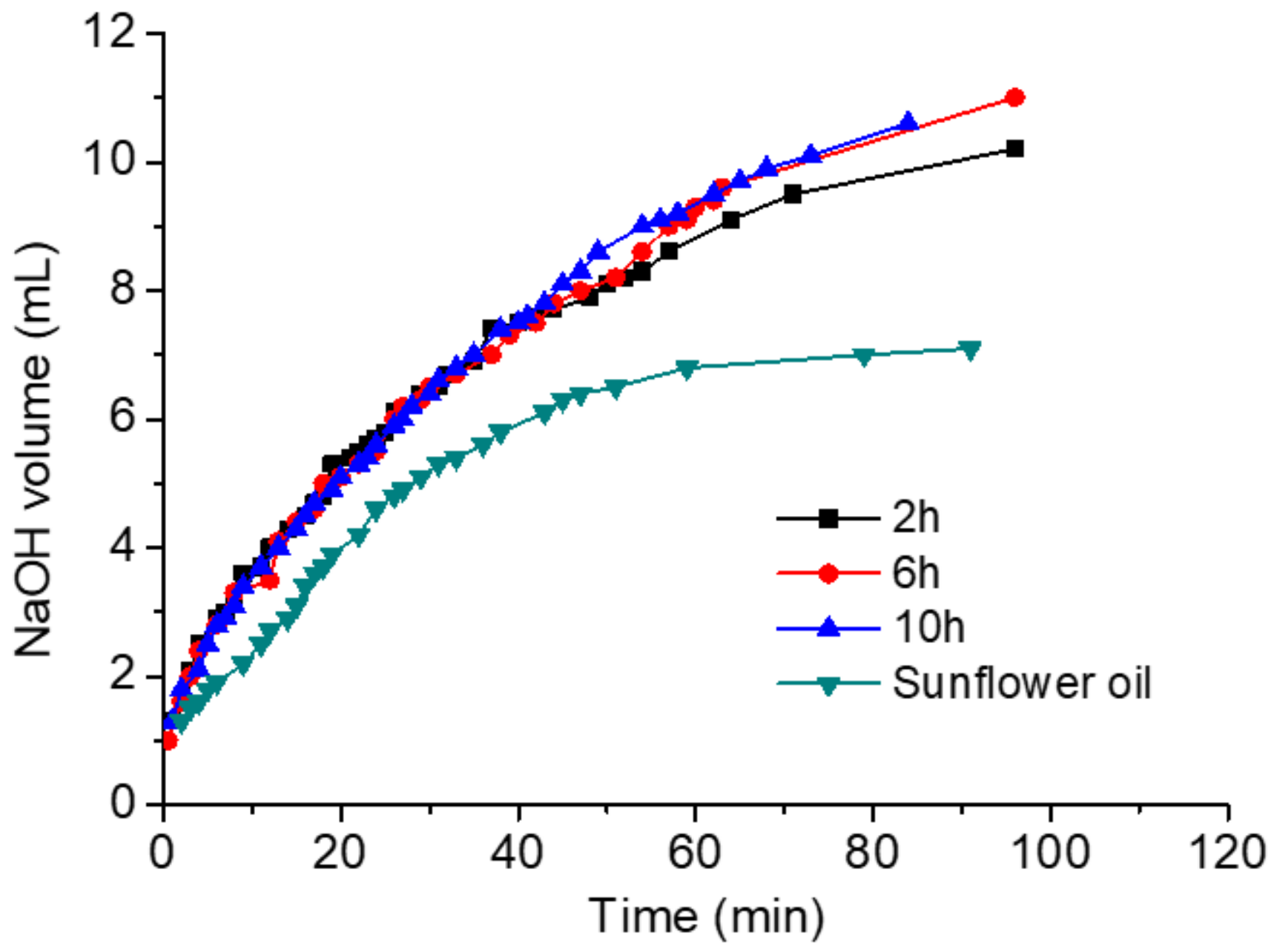

Figure 6

The influence of different nanoparticles on the free fatty acids release rate in the emulsions using the $\mathrm{pH}$ stat method was examined (Fig. 6). In general, there was a steep increase in the amount of free fatty acids released from the emulsions initially, followed by a more gradual increase at longer times. However, there were statistically significant differences between the rate and extent of digestion depending on the initial degree of dispersion of the oil droplets. In the case of the emulsions, the initial rate of digestion was much faster, and the final amount of free fatty acids released after $2 \mathrm{~h}$ of incubation was higher than for the pure sunflower oil. 


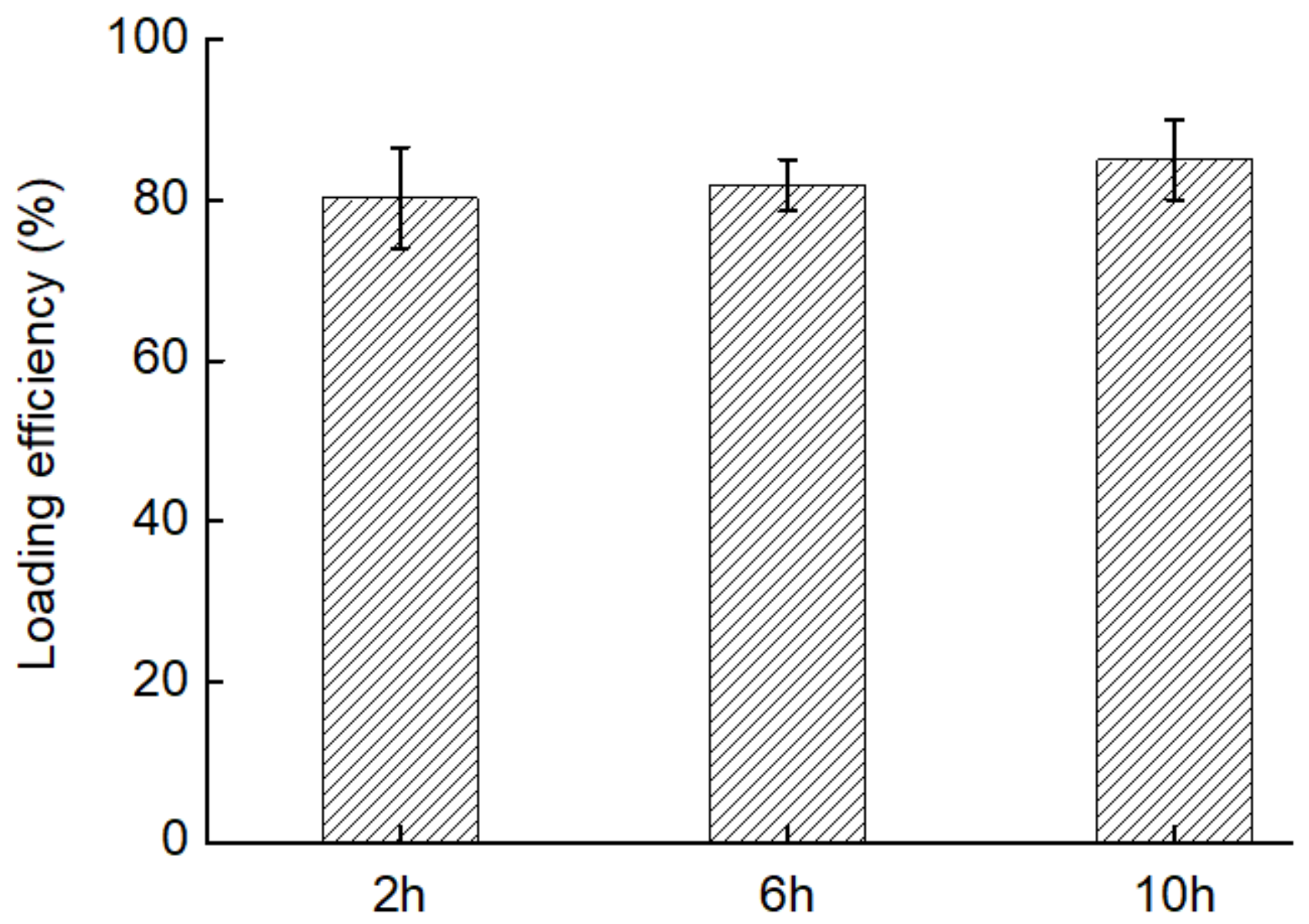

Figure 7

The application field was limited due to the volatility and instability of essential oil, consequently an appropriate emulsifier and formula are necessary to reduce the loss of functional material through embedding in the bilayer wall. Fig. 7 shows the effect of cellulose particle with different hydrolysis time on loading efficiency of thyme oil. When the hydrolysis time was $2 \mathrm{~h}$, the loading efficiency of thyme oil reached $79 \%$. The loading efficiency of thyme oil slightly increased as the hydrolysis time increased. 

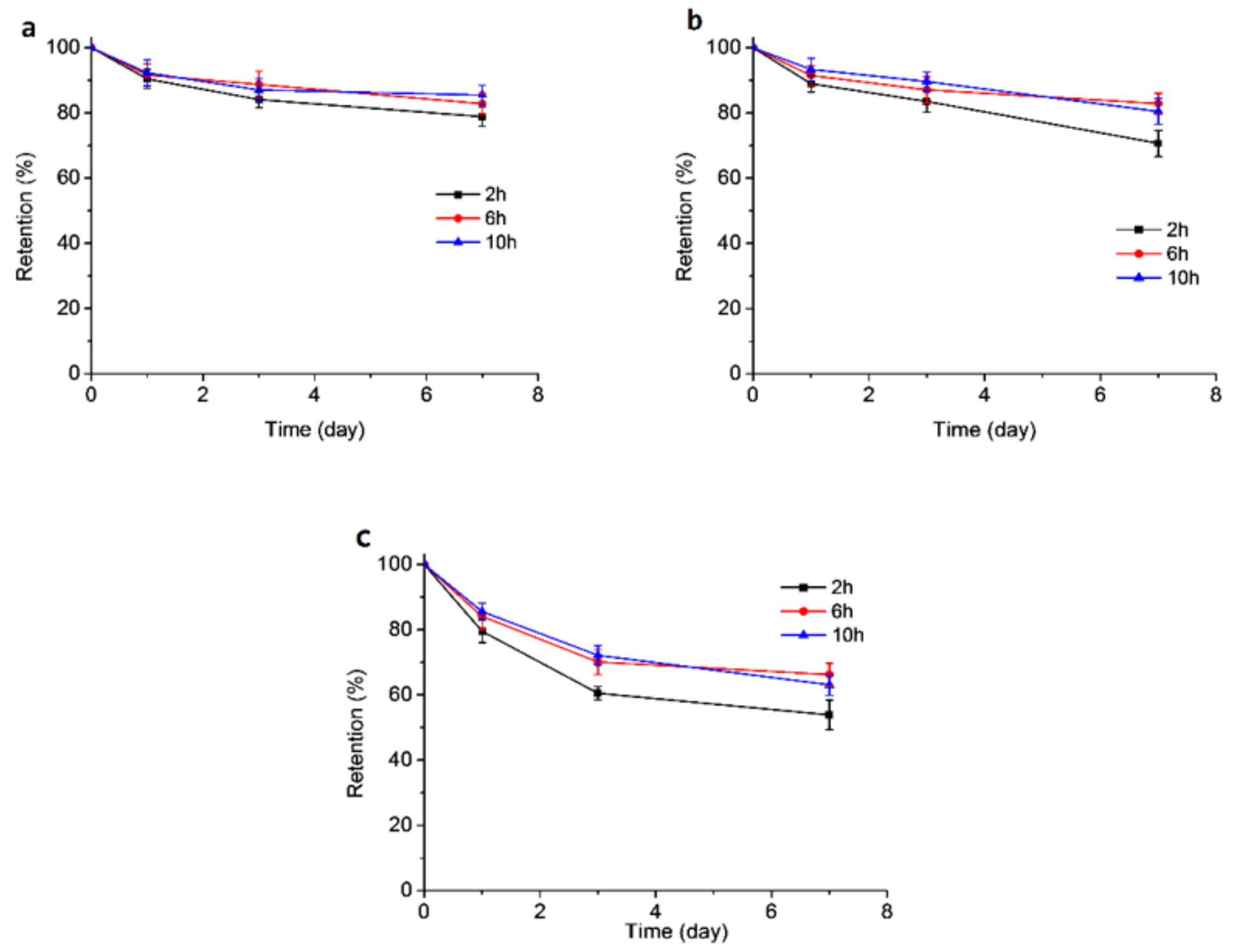

Figure 8

Thyme oil was a volatile essential oil substance, so the stability of thyme oil was an important parameter to measure the performance of emulsifier. 7 days storage was carried out to investigate the effect of temperature on the chemical stability of thyme oil emulsions (Fig. 8). The retention of thyme oil in samples at all the storage conditions decreased during the storage period. When the emulsion stored at $4 \mathrm{oC}$, the loss of thyme oil was less and the retention rate was still above $80 \%$ after 7 days. When the temperature rose to $25 \mathrm{oC}$ and $37 \mathrm{oC}$, the retention of thyme oil dropped to $70 \%$ and $55 \%$ for emulsion stabilized by cellulose $(2 \mathrm{~h})$, respectively.

\section{Supplementary Files}

This is a list of supplementary files associated with this preprint. Click to download.

- graphicalabstract.docx 